Research letter

\title{
Development of lymphocytes apoptosis at the first phase of acute pancreatitis
}

\author{
Svetlana S. Dunaevskaya, Daria A. Antufrieva \\ Voino-Yasenetsky Krasnoyarsk State Medical University, Krasnoyarsk, Russia \\ Received 10 April 2017, Revised 13 December 2017, Accepted 24 December 2017
}

(C) 2017, Dunaevskaya S.S., Antufrieva D.A.

(C) 2017, Russian Open Medical Journal

Abstract: Introduction - Acute pancreatitis takes the main place in the structure of surgical abdominal diseases. Endothelial dysfunction is one of the development mechanisms of the severe acute pancreatitis, which is accompanied by the formation of leucocytal and platelet microparticles, and massive cells apoptosis.

Aim - To study lymphocytes apoptosis depending on severity of acute pancreatitis and area of pancreas and retroperitoneal cellular tissue affection.

Material and Methods - We examined 40 patients with acute pancreatitis of IA phase of the disease, who were divided into two groups: with severe and non-severe course of the disease. Condition of lymphocytes plasma membrane in peripheral blood was estimated at admission of the patients by phase-contrast microscopy. Condition of pancreatic glandular tissue and retroperitoneal space were estimated by computed tomography angiography.

Results - In most cases, non-severe acute pancreatitis was not associated with development of necrotic affection of pancreatic glandular tissue. In the event of severe acute pancreatitis structural changes of the pancreatic glandular tissue and retroperitoneal cellular tissue were in $95 \%$ of the cases. In cases of severe acute pancreatitis, the total blebbing of lymphocytes in terms of 100 cells was 33.5 (27.1, 39.7), terminal - $21.9(14.6,28.3)$ (data presented as median with lower and upper quartiles). There were high positive correlations between the number of lymphocytes at terminal blebbing and affection in pancreatic gland tissue $(r=0.76, p<0.001)$, also terminal blebbing and retroperitoneal space affection $(r=0.82, p<0.001)$.

Conclusion - Lymphocytes apoptosis in peripheral blood reflects the severity of acute pancreatitis.

Keywords: acute pancreatitis, blebbing, apoptosis, lymphocytes

Cite as Dunaevskaya SS, Antufrieva DA. Development of lymphocytes apoptosis at the first phase of acute pancreatitis. Russian Open Medical Journal 2018; 7: e0103.

Correspondence to Svetlana S. Dunaevskaya. Address: 1, Partizana Zheleznyaka str., Krasnoyarsk, 660077, Russia. E -mail: vikto-potapenk@yandex.ru.

\section{Introduction}

Severe acute pancreatitis and its complications stay currently one of the important problems in modern surgery. Severe forms of the disease are diagnosed in approximately $15-25 \%$ of people with pancreatitis [1-3]. Release of a great number of biologically active compounds, which starts cascade of pathological reactions, plays leading role in pathogenesis of severe acute pancreatitis. The first phase of the disease is characterized by development of dangerous complications such as early multiple organ failure and shock [4-5].

Severe acute pancreatitis is associated with massive cells apoptosis, which is caused by oxidative stress [6]. Microparticles of leucocytal and platelet origin are the activators of endotheliocytes apoptosis [7]. Massive blebbing of lymphocytes plasma membrane is caused by oxidative stress due to necrosis of pancreatic glandular tissue. It shows indirectly the severity of acute pancreatitis [8].

Severe acute pancreatitis is accompanied by immunodeficiency and impairment cellular immunity due to peripheral lymphocytes apoptosis. All this leads to development of subsequent infectious complications of acute pancreatitis.

Aim of the study: to study lymphocytes apoptosis depending on severity of acute pancreatitis and area of pancreas and retroperitoneal cellular tissue affection.

\section{Material and Methods}

Forty patients with acute pancreatitis of IA phase of the disease underwent medical treatment at the Railway Hospital at Krasnoyarsk station of Russian Railways from 2015 to 2016.

Patients were divided into two groups: the $1^{\text {st }}$ group contained 20 patients with no severe acute pancreatitis; the $2^{\text {nd }}$ group had 20 patients with severe acute pancreatitis. We determined severity of acute pancreatitis in conformity with IAP/APA (International Association of Pancreatology / American Pancreatic Association) evidence-based guidelines for the management of acute pancreatitis [9-10]. The study was made in accordance with the Good Clinical Practice and principle of the Declaration of Helsinki. All participants signed informed consent before the study. 
Table 1. Pancreas affection in patients with acute pancreatitis

\begin{tabular}{|c|c|c|c|}
\hline Parameters & No severe acute pancreatitis, $n(M \pm m, \%)$ & Severe acute pancreatitis, $n(M \pm m, \%)$ & $p$-level (t-test) \\
\hline Interstitial edema of pancreatic gland tissue & $15(75.0 \pm 9.68 \%)$ & $1(5.0 \pm 4.87 \%)$ & $p<0.001$ \\
\hline Small-focal pancreatonecrosis ( $\mathrm{V}<30 \%)$ & $4(20.0 \pm 8.94 \%)$ & $7(35.0 \pm 10.67 \%)$ & $p=0.215$ \\
\hline Large-focal pancreatonecrosis ( $\mathrm{V}=30-50 \%)$ & $1(5.0 \pm 4.87 \%)$ & $8(40.0 \pm 10.95 \%)$ & $p=0.011$ \\
\hline Subtotal pancreatonecrosis (V=51-75\%) & $0(0 \%)$ & $3(15.0 \pm 7.98 \%)$ & - \\
\hline Total pancreatonecrosis ( $\mathrm{V} \geq 75 \%)$ & $0(0 \%)$ & $1(5.0 \pm 4.87 \%)$ & - \\
\hline
\end{tabular}

$\mathrm{V}$, volume; $n$, number; $\mathrm{P}$, percentage; $\mathrm{m}$, standard error of percentage.

Table 2. Retroperitoneal cellular tissue affection in patients with acute pancreatitis

\begin{tabular}{|c|c|c|c|}
\hline Parameters & No severe acute pancreatitis, $n(M \pm m, \%)$ & Severe acute pancreatitis, $n(M \pm m, \%)$ & $p$-level (t-test) \\
\hline Without cellular tissue affection & $16(80.0 \pm 8.94 \%)$ & $2(10.0 \pm 6.71 \%)$ & $p<0.001$ \\
\hline Affection of less than 3 regions & $3(15.0 \pm 7.98 \%)$ & $6(30.0 \pm 10.25 \%)$ & $p=0.307$ \\
\hline Affection of $4-6$ regions & $1(5.0 \pm 4.87 \%)$ & $8(40.0 \pm 10.95 \%)$ & $\mathrm{p}<0.001$ \\
\hline Affection of 7 and more regions & $0(0 \%)$ & $4(20.0 \pm 8.94 \%)$ & - \\
\hline
\end{tabular}

Table 3. Lymphocytes apoptosis in peripheral blood in cases of severe acute pancreatitis

\begin{tabular}{|c|c|c|c|}
\hline Parameters & No severe acute pancreatitis $(n=20)$ & Severe acute pancreatitis $(n=20)$ & p-level \\
\hline Lymphocytes at initial blebbing in terms of 100 cells & $6.8(5.4,9.0)$ & $13.4(12.6,15.3)$ & $p<0.001$ \\
\hline Lymphocytes at terminal blebbing in terms of 100 cells & $3.1(1.7,3.9)$ & $21.9(14.6,28.3)$ & $\mathrm{p}<0.001$ \\
\hline Total blebbing of lymphocytes in terms of 100 cells & $11.2(8.0,13.4)$ & $33.5(27.1,39.7)$ & $\mathrm{p}<0.001$ \\
\hline
\end{tabular}

Table 4. Correlation coefficients between lymphocytes apoptosis in peripheral blood and type of affection in pancreatic gland and retroperitoneal space

\begin{tabular}{lcc}
\hline Lymphocytes at initial blebbing in terms of 100 cells & Necrosis in pancreatic gland tissue & Retroperitoneal cellular tissue affection \\
Lymphocytes at terminal blebbing in terms of 100 cells & $r S=0.46, p<0.001$ & $r S=0.57, p<0.001$ \\
Total blebbing of lymphocytes in terms of 100 cells & $r S=0.76, p<0.001$ & $r S=0.82, p<0.001$ \\
\hline
\end{tabular}

$r S$, Spearman correlation coefficient; $p$, double tail significance.

Signs of pancreatic glandular tissue and retroperitoneal space affections were evaluated not earlier than the $14^{\text {th }}$ day of the disease with computed tomography (CT) angiography on multispiral 4 multislice tomograph «Lightspeed» (General Electric, USA). The pancreas affection was described by the relative value of pancreatonecrosis focus volume. By 3D-modelling we found out the volume of pancreatic gland $(\mathrm{ml})$, the volume of pancreatonecrosis $(\mathrm{ml})$, and we calculated relative pancreatonecrosis focus volume (\%). Retroperitoneal cellular tissue affection was estimated based on the number of anatomical regions of retroperitoneal cellular tissue with infiltration.

To estimate the activity of lymphocytes apoptosis in peripheral blood we took blood samples from the patients at the admission. The study was made at Research Institute of Molecular Medicine and Pathobiochemistry of Voino-Yasenetsky Krasnoyarsk State Medical University (Krasnoyarsk, Russia). Lymphocyte culture was selected by the standard method: centrifugation of heparinized blood by density gradient with Lympholyte-H CL5010 (Cedarlane Laboratories Limited, Canada). The plasma membrane condition was estimated by phase-contrast microscopy with microscope Olympus BX41 (Olympus Corp., Japan). We estimated such indicators as lymphocytes at initial blebbing (small vesicles less than $1 / 3$ of cell radius) in terms of 100 cells, lymphocytes at terminal blebbing (large vesicles more than $1 / 3$ of cell radius) in terms of 100 cells and total blebbing of lymphocytes (sum of the lymphocytes at initial blebbing and the lymphocytes at terminal blebbing) at more than 10 fields of view.

The obtained results were processed by statistical software package SPSS 17.0. The frequency and the character of the pancreas affection and the retroperitoneal cellular tissue affection are presented as absolute value with percentage and its standard error - $n(P \pm m \%)$. T-test for relative value was used for comparisons of studying groups. Based on the Shapiro-Wilk test, all examined parameters of the apoptosis did not correspond to the normal distribution. These data are presented as median with lower and upper quartiles - Me (LQ, UQ). Mann-Whitney $U$ test was used for pairwise comparisons of studying groups of patients. If $\mathrm{P}<0.05$, differences between groups were recognized as statistically significant. Association between parameters was revealed based on the Spearman correlation test (rS).

\section{Results}

The severity of acute pancreatitis depends on the area of pancreatic glandular tissue affection. Based on CT-angiography findings, interstitial edema of tissue without signals of necrotic affection dominated in cases of non-severe acute pancreatitis in $75.0 \pm 9.68 \%$ of cases, necrotic affection less than $30 \%$ of pancreas area was in $20.0 \pm 8.94 \%$ of cases. In case of severe acute pancreatitis necrotic affection of pancreatic glandular tissue was in $95.0 \pm 4.87 \%$, the domination of large-focal affection was in $40.0 \pm 10.95 \%$ of cases (Table 1 ).

In cases of no severe acute pancreatitis retroperitoneal cellular tissue was not involved in pathological processes in $80 \pm 8,94 \%$ of cases, in other cases, infiltration of retroperitoneal cellular tissue accompanied the formation of necrosis in pancreatic glandular tissue. In $90.0 \pm 6.71 \%$ cases of severe acute pancreatitis retroperitoneal cellular was involved in pathological processes. The vast affection of cellular tissue dominated, it included 4-6 anatomical regions (Table 2).

The severe acute pancreatitis was characterized by massive lymphocytes apoptosis in peripheral blood. The total blebbing of lymphocytes in terms of 100 cells was 33.5 (27.1, 39.7), it was 3 times more than the index in cases of no severe acute pancreatitis $(p<0.001)$. Number of cells at terminal blebbing in cases of severe acute pancreatitis was $21.9(14.6,28.3)$, in cases of no severe $-3.1(1.7,3.9)(p<0.001)($ Table 3$)$. 
The most interesting value was correlation between indicators of lymphocyte membrane apoptosis in peripheral blood and pathological changes at pancreatic glandular and retroperitoneal space. There were high positive correlations between the number of lymphocytes at terminal blebbing and necrosis in pancreatic gland tissue $(r S=0.76, p<0.001)$, also terminal blebbing and retroperitoneal space affection $(r S=0.82, p<0.001)$. Total blebbing of lymphocytes had moderate positive correlations with area of pancreas affection $(r S=0.68, p<0.001)$ and involvement of retroperitoneal cellular tissue in pathological processes $(r S=0.73$, $\mathrm{p}<0.001$ ) (Table 4).

\section{Discussion}

Development of severe acute pancreatitis is accompanied distinct oxidative stress, which causes the cascade of biochemical reactions, the base of pathogenesis of pancreatonecrosis [11]. In case of severe acute pancreatitis there is an endothelial dysfunction, it causes the increase of vessel walls permeability, impairment of hemostasis. Many authors connect development of severe complication, such as acute kidney injury and shock, with development of endothelial dysfunction [12]. Lymphocytes apoptosis in peripheral blood is non-specific marker, it shows intensity of functional condition of endothelium impairment.

Kataoka et al. (2004) showed how important to study role of apoptosis in severe acute pancreatitis and how hard to make a clinical study in this field [13]. The obtained data about the correlations between severity of acute pancreatitis and lymphocytes apoptosis intensity in peripheral blood coheres with multiple findings in this field, such as Qin et al. [14] findings and experimental findings by Pinhu et al. [15], and Weis et al. [16].

A distinctive feature of this study is high positive correlations between activity of lymphocyte apoptosis in peripheral blood and necrosis in pancreatic gland tissue, and infiltration of retroperitoneal cellular tissue, which was revealed by CTangiography.

\section{Conclusion}

The apoptosis due to the development of oxidative stress accompanies the severe clinical course of acute pancreatitis, destructive changes in pancreatic glandular and retroperitoneal cellular tissue. Lymphocytes apoptosis in peripheral blood reflects the severity of acute pancreatitis; it directly correlates with the area of pancreas and retroperitoneal cellular tissue affection.

\section{Acknowledgements}

The authors would like to thank the administration of Railway Hospital at Krasnoyarsk station of Russian Railways for the opportunity to conduct clinical research.

\section{Conflict of interest}

The authors of the article have no conflicts of interest to disclose. Authors of the article confirm the absence of sponsorship.

\section{Ethical approval}

All procedures performed in studies involving human participants were in accordance with the ethical standards of the institutional research committee and with the 1964 Helsinki declaration and its later amendments or comparable ethical standards.

\section{References}

1. Kim YJ, Kim DB, Chung WC, Lee JM, Youn GJ, Jung YD, et al. Analysis of factors influencing survival in patients with severe acute pancreatitis. Scand J Gastroenterol 2017; 7: https://dx.doi.org/10.1080/00365521.2017.1310291.

2. Kochetova LV, Dunayevskaya SS. Metabolic correction in combined treatment of patients with acute pancreatitis. Kazan Medical Journal 2011; 92(3): 315-318. Russian. https://elibrary.ru/item.asp?id=16443581.

3. Garcia-Hernandes V, Sarmiento N, Sanches-Bernal C, Matellán L, Calvo JJ, Sánchez-Yagüe J. Modulation in the expression of SHP-1, SHP-2 and PTP1B due to the inhibition of MARKs, cAMP and neutrophils early on in the development of cerulean-induced acute pancreatitis in rats. Biochim Biophys Acta 2014; 1842(2): 192-201. https://doi.org/10.1016/j.bbadis.2013.11.003.

4. Fomin AV, Gidranovich AV. Evaluation of severity of condition of patients with sharp pancreatitis (review). Vestnik Vitebskogo Gosudarstvennogo Meditsinskogo Universiteta 2004; (1): 41-50. Russian. https://elibrary.ru/item.asp?id=15218068.

5. Rathnakar SK, Vishnu VH, Muniyappa S, Prasath A. Accuracy and predictability of PANC-3 scoring system over APACHE II in acute pancreatitis: a prospective study. J Clin Diagn Res 2017; 11(2): 10-13. https://dx.doi.org/10.7860/JCDR/2017/23168.9375.

6. Soyalp $\mathrm{M}$, Yalcin $\mathrm{M}$, Oter $\mathrm{V}$, Ozgonul A. Investigation of procalcitonin, IL-6, oxidative stress index (OSI) plasma and tissue levels in experimental mild and severe pancreatitis in rats. Bratis/ Lek Listy 2017; 118(3): 137-141. https://doi.org/10.4149/BLL 2017027.

7. Russin VI, Sirchak ES, Kurchak NIU. Endothelial dysfunction at patients with chronic pancreatitis. Eksperimentalnaya $i$ Klinicheskaya Gastroenterologiya 2014; (5): 79. Russian. U https://elibrary.ru/item.asp?id=22569245.

8. Inzhutova Al, Salmina AB, Petrova MM, Mikhutkina SV, Narodova VV, Domrachev DV. Registration of plasma membrane blebbing of peripheral blood lymphocytes as an express method for assessment of severity of status in patients withypertension and stroke. Siberian Scientific Medical Journal 2007; 27(1): 6-10. Russian. https://elibrary.ru/item.asp?id=9444390.

9. Banks PA, Bollen TL, Dervenis C, Gooszen HG, Johnson CD, Sarr MG, et al. Classification of acute pancreatitis 2012: revision of the Atlanta classification and definitions by international consensus. Gut 2013; 62(1): 102-111. https://doi.org/10.1136/gutinl-2012-302779.

10. IAP/APA evidence-based guidelines for the management of acute pancreatitis. Working Group IAP/APA (International Association of Pancreatology/American Pancreatic Association) Acute Pancreatitis Guide-lines. Pancreatology 2013; 13: 1-15. https://doi.org/10.1016/j.pan.2013.07.063.

11. Horoshilov SE, Nikulin AV, Marukhov AV. The prevention of development of a syndrome of a multiorgan failure in an enzymatic phase of heavy sharp pancreatitis. Vestnik Natsionalnogo MedikoKhirurgicheskogo Tsentra im. N.I. Pirogova 2014; 9 (1): 58-62. Russian. https://elibrary.ru/item.asp?id=27285391.

12. Popov AV, Ershova Al, Podtaev SU. Investigation of the endotheliumdependent mechanism of microvascular tone regulation in patients with severe acute pancreatitis. Regionarnoe Krovoobrashchenie $i$ Mikrotsirkulyatsiya 2014; 13(4): 49-57. Russian. https://elibrary.ru/item.asp?id=22808036.

13. Kataoka K, Yasuda H, Sakagami J. Role of apoptosis in severe acute pancreatitis. Nihon Rinsho 2004; 62(11): 2021-2026. Japanese. https://www.ncbi.nlm.nih.gov/pubmed/15552884.

14. Qin $Y$, Liao $P, H e ~ S$, Yin $Y$, Song $S$, Hu J, et al. Detection of FasL mRNA, sFasL and their regulatory effect on $T$ lymphocyte subsets in patients with severe acute pancreatitis. Xi Bao Yu Fen Zi Mian Yi Xue Za Zhi 2013; 29(11): 11891192. Chinese. https://www.ncbi.nlm.nih.gov/pubmed/24200068.

15. Pinhu L, Qin Y, Xiong B, You Y, Li J, Sooranna SR. Overexpression of Fas and FasL is associated with infectious complications and severity of experimental severe acute pancreatitis by promoting apoptosis of 
lymphocytes. Inflammation 2014; 37(4): 12002-1212. https://doi.org/10.1007/s10753-014-9847-8.

16. Weis $S$, Schlaich $T$, Dehghani $F$, Carvalho $T$, Sommerer I, Fricke $S$, et al. p8 deficiency causes siderosis in spleens and lymphocyte apoptosis in acute pancreatitis. Pancreas 2014; 43(8): 1277-1285. https://doi.org/10.1097/MPA.0000000000000172.

Authors:

Svetlana S. Dunaevskaya - MD, Associate Professor, Department of General Surgery, Voino-Yasenetsky Krasnoyarsk State Medical University, Krasnoyarsk, Russia. http://orcid.org/0000-0003-2820-4737.

Daria A. Antufrieva - MD, Post-graduate student, Department of General Surgery, Voino-Yasenetsky Krasnoyarsk State Medical University, Krasnoyarsk, Russia. http://orcid.org/0000-0003-0190-7336. 